\title{
Outstanding Papers in Cellular and Molecular Bioengineering from the 2011 Biomedical Engineering Society Annual Meeting
}

The Biomedical Engineering Society (BMES) Annual Meeting in Hartford, Connecticut in October of 2011 was highly successful, with a strong set of contributions in the area of Cellular and Molecular Bioengineering.

To recognize excellence in the area of Cellular and Molecular Bioengineering, we, the Co-Editors-inChief, again dedicate a Special Issue of CMBE for recognition of the outstanding research presented at the BMES meeting. We thank the Cellular and Molecular Bioengineering Track reviewers, who evaluated all of the submitted abstracts and provided us with a scoring basis for selecting the most outstanding work. Also, we thank Cellular and Molecular Bioengineering Track Co-Chairs Kevin Costa (Mount Sinai School of Medicine) and Clark T. Hung (Columbia University), for their support of the Special Issue.

Contained in this issue are three of the most outstanding cellular and molecular bioengineering papers that were presented at the 2011 BMES meeting:

First, Richard Able, Jr., Maribel Vazquez, and co-workers at the City College of New York and Memorial Sloan Kettering Cancer Center, report their discovery that glial progenitor cells (GPCs) exhibit both chemoattraction and chemorepulsion by different concentrations of the same ligand (e.g. Transforming Growth Factor- $\alpha$ ). Since GPC recruitment is believed to facilitate glioma tumor growth, the results suggest novel ways in which GPCs might effectively be targeted for therapeutic benefit.

Second, Daniela Fuentes and Peter Butler at The Pennsylvania State University demonstrate that endothelial cells, which sense mechanical forces via focal adhesions (FAs), respond to applied force at a single FA by first recruiting lipid raft components and then later recruiting the adhesion-reinforcing component talin. To manipulate single FAs, the authors used a fibronectin-functionalized nanoelectrode with feedback control. They conclude that lipid raft recruitment is rapidly and directly responsive to mechanical force, and may facilitate subsequent recruitment of other FA components such as talin.

Third, Wylie Ahmed, Taher Saif, and co-workers at the University of Illinois-Urbana Champaign and the University of Miami, report that mechanical tension regulates synaptic vesicle accumulation and transport dynamics in Drosophila and Aplysia model systems, in vivo and in vitro, respectively. For example, in the case of their in vivo studies of neuromuscular junction synaptic vesicles, sustained stretch leads to sustained vesicle accumulation. The results suggest that mechanical tension may be used to promote synaptic activity in the nervous system.

We hope that you will enjoy reading these exciting Outstanding Papers from the 2011 BMES Meeting.

\author{
X. Edward Guo \\ Co-Editor-in-Chief \\ Columbia University \\ DAvid J. OdDE \\ Co-Editor-in-Chief \\ University of Minnesota \\ Electronic mail: oddex002@umn.edu
}

